Preface

\title{
Recent Advances in Thrombosis and Hemostasis-Part V
}

\author{
Sam Schulman, MD, $\mathrm{PhD}^{1,2}$ \\ 1 Department of Medicine, Thrombosis and Atherosclerosis Research \\ Institute, McMaster University, Hamilton, Ontario, Canada \\ 2 Department of Obstetrics and Gynecology, I.M. Sechenov First \\ Moscow State Medical University, Moscow, Russia
}

Semin Thromb Hemost 2019;45:757-759.

"One of the big questions in the climate debate: Are humans smarter than frogs in a pot?

If you put a frog in a pot and slowly turn up the heat, it won't jump out.

Instead it will enjoy the nice warm bath until it is cooked to death.

We humans seem to be doing pretty much the same thing."

Jeff Goodell, American author

In the fifth theme issue in the Recent Advances in Thrombosis and Hemostasis series, the reader will view a true variety of interesting presentations. Thrombosis and hemostasis are expanding areas, with an increasing number of identified connections with the immune system and inflammatory mechanisms. The previous issues in this series have mainly included papers on venous thromboembolism (VTE) and, to a smaller extent, on atrial fibrillation. The thrombi formed in atrial fibrillation originate in a low flow area-the atrial appendage-and are therefore actually similar to the venous thrombi.

The first contribution in this issue relates to the introductory quote. Ohbe et al studied 1,606 patients who had suffered heatstroke and developed disseminated intravascular coagulation (DIC) during a 7-year period in Japan. ${ }^{1}$ The focus of their study was the subset of patients who had been treated with one or both of the natural anticoagulants antithrombin and thrombomodulin, and the primary outcome was in-hospital mortality in comparison with patients without anticoagulant intervention. Unfortunately, we are likely to see an increasing number of patients with this condition, and with a mortality of $20 \%$, when complicated by DIC, there is certainly room for improvement.

A condition with even higher mortality rates is venoocclusive disease/sinusoidal obstruction syndrome, which

Address for correspondence Sam Schulman, MD, PhD, Thrombosis Service, HHS-General Hospital, 237 Barton Street East. Hamilton, Ontario L $8 \mathrm{~L} 2 \times 2$,

Canada

(e-mail: schulms@mcmaster.ca).

can occur after hematopoietic stem cell transplantation. In a systematic review and meta-analysis, Yang et al have analyzed the survival rate at 100 days after the transplant, as well as complete response, hemorrhagic events, and serious adverse events in patients with veno-occlusive disease and whether defibrotide, a complex compound of oligodeoxyribonucleotides, improves the results. ${ }^{2}$ Their findings should be regarded as hypothesis-generating since none of the studies had a randomized placebo-control design.

The theme issue then moves over to more traditional VTE. Thrombi in the anatomical region of the lungs are usually considered as emboli that have originated in veins elsewhere. Marongiu et al bring forward arguments that some of these events represent true pulmonary thrombosis as a result of local inflammatory disease and/or interplay with the innate immune system. ${ }^{3}$ They discuss the potential mechanisms for this occurrence in patients with pneumonia, chronic obstructive pulmonary disease, asthma, sickle cell disease, and Gaucher's disease. The increased risk for venous thrombosis in these disease entities may influence our decision to provide thromboprophylaxis.

Continuing on the theme of pulmonary embolism, Lippi et al have reviewed the utility of high-sensitivity cardiac troponin measurement for risk stratification in pulmonary embolism. ${ }^{4}$ This biomarker may provide additive value to the pulmonary embolism severity index. Whether the use of biomarkers to direct the choice of therapy makes a difference in clinical outcomes remains, however, to be seen.

The balance between bleeding and thrombosis in patients with liver cirrhosis is difficult to assess. Promoting factors for bleeding are thrombocytopenia, hypoprothrombinemia, and esophageal varices, whereas factors increasing the risk for thrombosis are the reduced levels of natural anticoagulants and stasis in the splanchnic veins. Bikdeli et al have used the large RIETE registry (Registro Informatizado de la Enfermedad TromboEmbolica), which includes patients with VTE, to compare outcomes among 187 cases with liver cirrhosis versus those without cirrhosis. ${ }^{5}$ The

Copyright $\odot 2019$ by Thieme Medical Publishers, Inc., 333 Seventh Avenue, New York, NY 10001, USA. Tel: +1(212) 584-4662.
DOI https://doi.org/

10.1055/s-0039-1700490. ISSN 0094-6176.
Thrombosis and Hemostasis-

Part V; Guest Editor: Sam

Schulman, MD, PhD. 
primary outcome was 30-day mortality, and, in addition, they analyzed major and clinically relevant nonmajor hemorrhage and recurrent VTE up to 90 days.

Thrombocytopenia is no guaranteed protection against thrombosis, and a wealth of data indicate that platelets play many roles in thrombus formation not only via platelet aggregation and as a surface for localized activation of coagulation factors but also via proinflammatory characteristics. This is reviewed by Stevens and McFadyen. ${ }^{6}$ The latter was an Eberhard F. Mammen Young Investigator Award winner in 2018, and this review article also represents a contribution according to the award conditions for such awardees.

Hereafter, the theme issue moves over to prophylaxis against VTE. Although a large number of meta-analyses of low-molecular-weight heparin in the prevention of VTE have been published, the one performed by Eck et al adds a trial sequential analysis. ${ }^{7}$ The authors compare this method to an interim analysis for an individual randomized trial, contributing an adjusted threshold for statistical significance in the cumulative meta-analysis. The authors included 70 randomized trials, and the outcomes were allcause mortality, symptomatic VTE, and major bleeding. As was already known, the authors find here that low-molecular-weight heparin reduces VTE but increases the risk of bleeding.

Statins have anti-inflammatory potential, and perhaps through this mechanism and/or via direct effects on the hemostatic system, with decreased levels of coagulation factors, the drug also appears to provide prevention against VTE. In this case, there is no evidence that statins increase the risk of bleeding. Here, Orsi et al have reviewed the different modes of action of statins and their effects in animal models of thrombosis and in primary prevention as well as secondary prophylaxis in humans. ${ }^{8}$ There is still a lack of interventional studies, and we are thus not yet ready to introduce statins for VTE prophylaxis in clinical practice. In a commentary to this article, Lippi and Favaloro add further thoughts to the extrapolation of statin use from the arterial side to VTE. ${ }^{9}$ They also discuss aspects of drug safety and health economy.

On a similar topic, Jiménez-Serranía and Lobato present a study on adverse drug reactions in patients on anticoagulants with or without statin medication and how they were able to pick up signals of drug reactions when combined with other medications such as analgesics, nonsteroidal antiinflammatory drugs, and proton pump inhibitors. ${ }^{10}$ The data source was the reporting from a network of 100 pharmacies in a region in Spain. The study highlights how we need to be aware of drug-drug interactions, more so with the increasing number of prescribed medications.

Another contribution that highlights how adding a drug to reduce risk of bleeding can cause an unwanted interaction is the article on proton pump inhibitors and dabigatran by Bolek et al. ${ }^{11}$ Their study adds evidence to previous results from phase II and phase III clinical trials that the combination leads to reduced plasma levels of dabigatran. It is, however, not entirely clear if this interaction is of clinical significance.

Patients with severe renal impairment are at increased risk of bleeding and thromboembolism, and therefore anticoagulant therapy is problematic. Spirk et al used a Swiss registry of 2,062 patients with VTE, of whom 240 had severe kidney disease, to compare management and 90-day clinical outcomes. ${ }^{12}$ Among their findings, acute or chronic comorbidities seemed to be important for the prognosis regarding 90-day survival, whereas the extent of the VTE of recurrent VTE was not.

The final contribution provided as correspondence deals with the use of antithrombin concentrate for acquired deficiency of the natural inhibitor and while on treatment with unfractionated heparin. The possibility that substitution with antithrombin can facilitate achievement of therapeutic heparin levels and reduce the amount of heparin given is evaluated by Arachchillage et al. ${ }^{13}$ They analyzed retrospectively 35 children with mechanical heart valve replacement, extracorporeal membrane oxygenation, or chylothorax, the latter two with or without concomitant venous or arterial thrombosis. These are obviously very ill children, and optimization of their treatment is paramount.

It is always an educational experience to work on and assemble a new theme issue. Therefore, hopefully, some or most of the contributions to this issue will also be of interest to the reader.

\section{Conflicts of Interest}

None.

\section{References}

1 Ohbe H, Isogai S, Jo T, Matsui H, Fushimi K, Yasunaga H. Treatment with antithrombin or thrombomodulin and mortality from heatstroke-induced disseminated intravascular coagulation: a nationwide observational study. Semin Thromb Hemost 2019;45(08): 760-766

2 Yang L, Qi J, Pan T, You T, Ruan C, Han Y. Efficacy and safety of defibrotide for the treatment of hepatic veno-occlusive disease after hematopoietic stem cell transplantation: a systematic review and meta-analysis. Semin Thromb Hemost 2019;45(08): 767-777

3 Marongiu F, Mameli A, Grandone E, Barcellona D. Pulmonary thrombosis: a clinical pathologic entity distinct from pulmonary embolism? Semin Thromb Hemost 2019;45(08):778-783

4 Lippi G, Favaloro EJ, Kavsak P. Measurement of high-sensitivity cardiac troponin in pulmonary embolism: useful test or a clinical distraction? Semin Thromb Hemost 2019;45(08):784-792

5 Bikdeli B, Jiménez D, Garcia-Tsao G, et al. Venous thromboembolism in patients with liver cirrhosis: findings from the RIETE registry. Semin Thromb Hemost 2019;45(08):793-801

6 Stevens H, McFadyen JD. Platelets as central actors in thrombosis reprising an old role and defining a new character. Semin Thromb Hemost 2019;45(08):802-809

7 Eck RJ, Bult W, Wetterslev J, et al. Intermediate dose low-molecular-weight heparin for thrombosis prophylaxis: systematic review with meta-analysis and trial sequential analysis. Semin Thromb Hemost 2019;45(08):810-824

8 Orsi FA, Cannegieter SC, Lijfering WM. Statin therapy to revert hypercoagulability and prevent venous thromboembolism: a narrative review. Semin Thromb Hemost 2019;45(08):825-833 
9 Lippi G, Favaloro EJ. Statins for preventing venous thrombosis: for or against? Semin Thromb Hemost 2019;45(08):834-836

10 Jiménez-Serranía M-I, Lobato CT. Influence of concomitant treatments under anticoagulants and statins in detecting signals of adverse drug reactions. Semin Thromb Hemost 2019;45(08):837-845

11 Bolek T, Samoš M, Škorňová I, et al. Proton pump inhibitors and dabigatran therapy: impact on gastric bleeding and dabigatran plasma levels. Semin Thromb Hemost 2019;45(08):846-850
12 Spirk D, Sebastian T, Banyai M, et al. Venous thromboembolism and renal impairment: insights from the SWIss Venous ThromboEmbolism Registry (SWIVTER). Semin Thromb Hemost 2019; 45(08):851-858

13 Arachchillage DRJ, Gaspar M, Makhecha S, Laffan M. Use of antithrombin concentrate for acquired antithrombin deficiency in acutely unwell children receiving unfractionated heparin. Semin Thromb Hemost 2019;45(08):859-864 